\title{
Synthesis of Carbazoles from Ynamides by Intramolecular Dehydro Diels-Alder Reactions
}

\author{
María Fernanda Martínez-Esperón, David Rodríguez, Luis Castedo, Carlos Saá* \\ Departamento de Química Orgánica y Unidad Asociada al CSIC, Facultad de Química, \\ Universidad de Santiago de Compostela,15782 Santiago de Compostela, Spain
}

\section{Supporting Information}

\section{Table of contents}

$\begin{array}{ll}\text { Experimental section } & \text { S2 }\end{array}$

$\begin{array}{ll}\text { General details } & \text { S2 }\end{array}$

$\begin{array}{ll}\text { A typical procedure and data: Ynamides } & \text { S2 }\end{array}$

$\begin{array}{ll}\text { A typical procedure and data:Thermal cyclizations } & \text { S5 }\end{array}$

$\begin{array}{lr}\text { Spectra } & \text { S9 }\end{array}$ 


\section{Experimental section}

\section{General details}

All reactions were carried out under argon atmosphere with magnetic stirring. The solvents were purified and dried using standard procedures. All reagents were purchased and used without further purification. ${ }^{1} \mathrm{H} \mathrm{NMR}$ at $250,13 \mathrm{MHz}$ and ${ }^{13} \mathrm{C}$ NMR spectra at $62,89 \mathrm{MHz}$ were determined using $\mathrm{CDCl}_{3}$ as solvent with tetramethylsilane as internal standard. Mass spectra were measured by ionizing the sample at $70 \mathrm{eV}$. Column chromatographies were made on silica gel 230-240 mesh (flash).

\section{Starting materials: General procedure}

Ynamides: To a stirred solution of N-tosylaniline $5(1 \mathrm{mmol})$ in dry DMF (20 mL) was added $\mathrm{Cs}_{2} \mathrm{CO}_{3}(1.3 \mathrm{eq})$ at $\mathrm{rt}$. After $30 \mathrm{~min}$, a solution of $\mathbf{6}(1.3 \mathrm{mmol})$ in dry $\mathrm{CH}_{2} \mathrm{Cl}_{2}(8$ $\mathrm{mL})$ was added dropwise. Stirring was continued until starting materials disappeared (TLC monitoring, typically $5 \mathrm{~h})$. Then, ether was added $(10 \mathrm{~mL})$ and the combined organic layers were extracted with water and brine, dried over anhydrous $\mathrm{Na}_{2} \mathrm{SO}_{4}$ and concentrated to dryness. Purification of the residue by flash chromatography on silica gel using a gradient mixture of EtOAc/hexanes as eluent afforded ynamides $\mathbf{1}$ in good yields.<smiles>C#CN(C)c1ccccc1C#C/C=C\C</smiles>

Clear oil; 68\% yield; ${ }^{1} \mathrm{H}$ NMR $\left(\mathrm{CDCl}_{3}\right) \delta: 7.71(\mathrm{~d}, J=8.3 \mathrm{~Hz}, 2 \mathrm{H}, \mathrm{ArH}), 7.47-7.44(\mathrm{~m}$, $1 \mathrm{H}, \operatorname{ArH}), 7.35-7.21(\mathrm{~m}, 5 \mathrm{H}, \mathrm{ArH}), 6.07-5.94(\mathrm{~m}, 1 \mathrm{H}, \mathrm{C}=\mathrm{CH}), 5.47$ (dd, J=10.7, 1.6 $\mathrm{Hz}, 1 \mathrm{H}, \mathrm{C}=\mathrm{CH}), 3.83(\mathrm{~s}, 1 \mathrm{H}, \mathrm{C} \equiv \mathrm{CH}), 2.41\left(\mathrm{~s}, 3 \mathrm{H}, \mathrm{CH}_{3}\right), 1.90(\mathrm{dd}, J=6.9,1.6 \mathrm{~Hz}, 3 \mathrm{H}$, $\left.\mathrm{CH}_{3}\right) ;{ }^{13} \mathrm{C} \mathrm{NMR/DEPT}\left(\mathrm{CDCl}_{3}\right) \delta: 139.4(\mathrm{CH}), 137.8(\mathrm{C}), 134.2(\mathrm{C}), 133.4(\mathrm{CH}), 129.5$ $(2 \times C H), 129.1(2 \times C H), 128.7(C H), 128.3(2 \times C H), 123.5(C), 110.4(C), 109.7$ $(C \mathrm{H}), 92.2(C), 89.0(C), 75.8(C H), 58.6(C), 21.6\left(C_{3}\right), 16.2\left(C_{3}\right) ;$ MS $(70 \mathrm{eV}) \mathrm{m} / \mathrm{z}$ (\%): $335\left(\mathrm{M}^{+}, 2\right), 156(31), 123$ (51), 91 (100); HRMS $\left(\mathrm{C}_{20} \mathrm{H}_{17} \mathrm{NO}_{2} \mathrm{~S}\right)$ : calc.: 335.0980, found: 335.0979 .

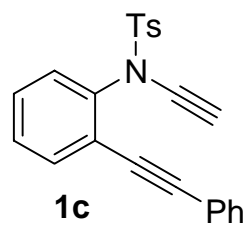

Clear oil, 77\% yield; ${ }^{1} \mathrm{H}$ NMR $\left(\mathrm{CDCl}_{3}\right) \delta: 7.68(\mathrm{~d}, J=7.8 \mathrm{~Hz}, 2 \mathrm{H}, \mathrm{ArH}), 7.51-7.30(\mathrm{~m}$, 9H, $\mathrm{ArH}), 7.07$ (d, J= 7.8 Hz, 2H, $\mathrm{ArH}), 2.94(\mathrm{~s}, 1 \mathrm{H}, \mathrm{C} \equiv \mathrm{CH}), 2.15\left(\mathrm{~s}, 3 \mathrm{H}, \mathrm{CH}_{3}\right) ;{ }^{13} \mathrm{C}$ 
NMR/DEPT $\left(\mathrm{CDCl}_{3}\right) \delta: 144.9(C), 138.0(C), 134.2(C), 133.1(C \mathrm{H}), 131.4(2 \times C \mathrm{x})$, $129.7(\mathrm{CH}), 129.5(2 \times \mathrm{CH}), 129.1(\mathrm{CH}), 128.9(\mathrm{CH}), 128.4(\mathrm{CH}), 128.1(2 \times \mathrm{CH})$, $127.9(2 \times C H), 122.6(C), 122.5(C), 95.3(C), 84.6(C), 75.6(C H), 59.1(C), 21.3$ $\left(\mathrm{CH}_{3}\right)$; MS (FAB) $\mathrm{m} / z(\%): 372\left(\mathrm{M}^{+}+1,3\right), 231$ (61), 154 879), 137 (100); HRMS FAB $\left(\mathrm{C}_{23} \mathrm{H}_{17} \mathrm{NO}_{2} \mathrm{~S}\right)$ : calc.: $(\mathrm{M}+1) 372.1058$, found: 372.1052 .<smiles>C#CN(C)c1ccccc1C#Cc1cccc2ccccc12</smiles>

Clear oil, 25\% yield; ${ }^{1} \mathrm{H}$ NMR $\left(\mathrm{CDCl}_{3}\right) \delta: 8.35-8.31(\mathrm{~m}, 1 \mathrm{H}, \mathrm{ArH}), 7.84(\mathrm{~d}, J=7.9 \mathrm{~Hz}$, 2H, ArH), 7.69-7.39 (m, 10H, ArH), $6.87(\mathrm{~d}, J=7.9 \mathrm{~Hz}, 2 \mathrm{H}, \mathrm{ArH}), 3.02(\mathrm{~s}, 1 \mathrm{H}, \mathrm{C} \equiv \mathrm{CH})$, $1.83\left(\mathrm{~s}, 3 \mathrm{H}, \mathrm{CH}_{3}\right) ;{ }^{13} \mathrm{C}$ NMR/DEPT $\left(\mathrm{CDCl}_{3}\right) \delta: 144.9(\mathrm{C}), 138.1(\mathrm{C}), 134.3(\mathrm{C}), 133.6$ $(C \mathrm{H}), 132.94(C), 132.89(C), 130.7(C H), 130.1(C H), 129.4(2 \times C H), 129.3(C H)$, $129.2(\mathrm{CH}), 129.0(\mathrm{CH}), 128.2(2 \times C H), 128.0(\mathrm{CH}), 126.7(\mathrm{CH}), 126.6(C \mathrm{H}), 126.4$ $(C \mathrm{H}), 125.0(C \mathrm{H}), 122.9(C), 120.5(C), 93.4(C), 89,4(C), 76.1(C \mathrm{H}), 59.1(C), 21.1$ $\left(\mathrm{CH}_{3}\right)$; MS (FAB) $\mathrm{m} / z(\%): 422\left(\mathrm{M}^{+}+1,5\right), 301$ (13), 282 (100); HRMS FAB $\left(\mathrm{C}_{27} \mathrm{H}_{19} \mathrm{NO}_{2} \mathrm{~S}\right)$ : calc. $(\mathrm{M}+1)$ : 422.1214 , found: 422.1212 .<smiles>C#CN(C)c1ccccc1C#Cc1cc2ccccc2c2ccccc12</smiles>

Clear oil, $69 \%$ yield; ${ }^{1} \mathrm{H}$ NMR $\left(\mathrm{CDCl}_{3}\right) \delta: 8.71-8.67(\mathrm{~m}, 3 \mathrm{H}, \mathrm{Ar} H), 8.44(\mathrm{~d}, J=7.5 \mathrm{~Hz}$, 1H, $\operatorname{ArH}), 7.87-7.85(\mathrm{~m}, 2 \mathrm{H}, \operatorname{ArH}), 7.72-7.63(\mathrm{~m}, 6 \mathrm{H}, \mathrm{ArH}), 7.47-7.43$ (m, 3H, ArH), $6.86(d, J=8.0 \mathrm{~Hz}, 2 \mathrm{H}, \mathrm{ArH}), 3.05(\mathrm{~s}, 1 \mathrm{H}, \mathrm{C} \equiv \mathrm{CH}), 1.74\left(\mathrm{~s}, 3 \mathrm{H}, \mathrm{CH}_{3}\right) ;{ }^{13} \mathrm{C}$ NMR/DEPT $\left(\mathrm{CDCl}_{3}\right) \delta: 144.9(C), 138.2(C), 134.3(C), 133.6(C \mathrm{H}), 132.2(C \mathrm{H}), 130.9(C), 130.6$ $(C), 130.2(C), 130.0(C H), 129.7(C), 129.34(2 \times C H), 129.28(C H), 129.2(C H)$, $128.5(\mathrm{CH}), 128.2(2 \times \mathrm{CH}), 127.6(\mathrm{CH}), 127.3(\mathrm{CH}), 127.0(\mathrm{CH}), 126.9(2 \times \mathrm{CH})$, $122.8(C), 122.5(C H), 122.4(C H), 119.2(C), 93.6(C), 89.1(C), 76.2(C H), 59.2(C)$, $20.9\left(\mathrm{CH}_{3}\right)$; MS (FAB) $\mathrm{m} / z(\%): 472\left(\mathrm{M}^{+}+1,13\right), 293$ (100), 137 (67); HRMS FAB $\left(\mathrm{C}_{31} \mathrm{H}_{21} \mathrm{NO}_{2} \mathrm{~S}\right)$ : calc. $(\mathrm{M}+1)$ : 472.1371 , found: 472.1369 . 


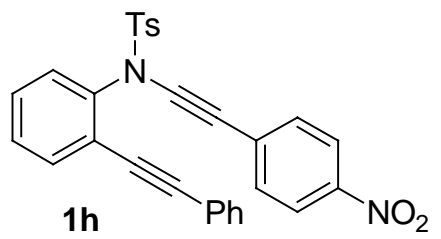

Clear oil; ${ }^{1} \mathrm{H}$ NMR $\left(\mathrm{CDCl}_{3}\right) \delta: 8.10(\mathrm{~d}, J=8.5 \mathrm{~Hz}, 2 \mathrm{H}, \mathrm{ArH}), 7.73(\mathrm{~d}, J=8.5 \mathrm{~Hz}, 2 \mathrm{H}$, $\mathrm{ArH})$, 7.57-7.52 (m, 2H, ArH), 7.46-7.41 (m, 4H, ArH), 7.28-7.10 (m, 7H, ArH), 2.18 $\left(\mathrm{s}, 3 \mathrm{H} \mathrm{CH} \mathrm{CH}_{3}\right) ;{ }^{13} \mathrm{C}$ NMR/DEPT $\left(\mathrm{CDCl}_{3}\right) \delta: 146.1(\mathrm{C}), 145.3(\mathrm{C}), 138.0(\mathrm{C}), 134.0(\mathrm{C})$, $133.4(\mathrm{CH}), 131.3(2 \times C \mathrm{H}), 130.8(2 \times C \mathrm{H}), 130.3(C), 129.9(\mathrm{CH}), 129.7(2 \times C \mathrm{H})$, $129.3(\mathrm{CH}), 129.1(\mathrm{CH}), 128.6(\mathrm{CH}), 128.2(2 \times \mathrm{CH}), 127.9(2 \times \mathrm{CH}), 123.5(2 \times \mathrm{CH})$, $122.4(C), 122.3(C), 95.6(C), 88.4(C), 84.4(C), 70.6(C), 21.4\left(C_{3}\right) ; \mathrm{MS}(70 \mathrm{eV}) \mathrm{m} / \mathrm{z}$ (\%): $492\left(\mathrm{M}^{+}, 29\right), 337$ (100), 281 (59); HRMS $\left(\mathrm{C}_{29} \mathrm{H}_{20} \mathrm{~N}_{2} \mathrm{O}_{4} \mathrm{~S}\right)$ : calc.: 492.1143, found: 492.1127.<smiles>CC#CN(C)c1ccccc1C#CC1=CCCCC1</smiles>

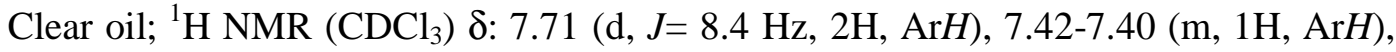
7.31-727 (m, 5H, $\mathrm{ArH}), 6.1(\mathrm{~s}, 1 \mathrm{H}, \mathrm{C}=\mathrm{CH}), 2.43$ (s, 3H, $\left.\mathrm{CH}_{3}\right), 2.09-2.08$ (bs, $2 \mathrm{H}, \mathrm{CH}_{2}$ ), 2.02-2.01 (bs, 2H, CH $), 1.62-1.58\left(\mathrm{~m}, 4 \mathrm{H}, \mathrm{CH}_{2}\right), 0.13\left(\mathrm{~s}, 3 \mathrm{H}, \mathrm{Si}\left(\mathrm{CH}_{3}\right)_{3}\right) ;{ }^{13} \mathrm{C}$ NMR/DEPT $\left(\mathrm{CDCl}_{3}\right) \delta: 144.5(C), 138.2(C), 135.9(C \mathrm{H}), 134.6(C), 133.2(C \mathrm{H}), 129.3$ (3 x $C H), 128.9(C H), 128.7(2 \times C H), 128.3(C H), 123.4(C), 120.5(C), 97.4(C), 94.4$ $(C), 82.4(C), 72.8(C), 28.7\left(C_{2}\right), 25.7\left(C_{2}\right), 22.2\left(C \mathrm{H}_{2}\right), 21.7\left(C_{3}\right), 21.5\left(C \mathrm{H}_{2}\right), 0.0$ $\left(\mathrm{Si}\left(\mathrm{CH}_{3}\right)_{3}\right)$.<smiles>C#CN([TeH])c1ccncc1C#Cc1ccccc1</smiles>

Clear oíl, $12 \%$ yield; ${ }^{1} \mathrm{H}$ NMR $\left(\mathrm{CDCl}_{3}\right) \delta: 8.75(\mathrm{~s}, 1 \mathrm{H}, \operatorname{Ar} H), 8.59(\mathrm{~d}, J=5.3 \mathrm{~Hz}, 1 \mathrm{H}$, $\operatorname{ArH}), 7.69$ (d, $J=8.1 \mathrm{~Hz}, 2 \mathrm{H}, \operatorname{ArH}), 7.42-7.27(\mathrm{~m}, 6 \mathrm{H}, \operatorname{ArH}), 7.13(\mathrm{~d}, J=8.1 \mathrm{~Hz}, 2 \mathrm{H}$, $\mathrm{ArH}), 2.99(\mathrm{~s}, 1 \mathrm{H}, \mathrm{C} \equiv \mathrm{CH}), 2.20\left(\mathrm{~s}, 3 \mathrm{H}, \mathrm{CH}_{3}\right) ;{ }^{13} \mathrm{C}$ NMR/DEPT $\left(\mathrm{CDCl}_{3}\right) \delta: 154.3(\mathrm{CH})$, $149.4(C \mathrm{H}), 145.5(C), 145.2(C), 133.9(C), 131.5(2 \times C H), 129.8(2 \times C H), 129.1$ $(\mathrm{CH}), 128.2(2 \times \mathrm{CH}), 128.1(2 \times \mathrm{CH}), 123.5(\mathrm{CH}), 122.1(C), 118.7(C), 98.9(C), 81.7$ (C), $74.4(\mathrm{CH}), 60.6(C), 21.5\left(\mathrm{CH}_{3}\right)$; MS (70 eV) m/z (\%): $372\left(\mathrm{M}^{+}, 2\right), 308(59), 217$ (100), 190 (18); HRMS $\left(\mathrm{C}_{22} \mathrm{H}_{16} \mathrm{~N}_{2} \mathrm{O}_{2} \mathrm{~S}\right)$ : calc.: 372.0925, found: 372.0932. 


\section{Thermal cyclizations: General procedures}

Conditions A: A toluene solution of $1(0.01 \mathrm{M})$ was placed in a sealed tube and heated overnight at $150{ }^{\circ} \mathrm{C}$ in a silicon oil bath. After evaporation of the solvent, the crude material was purified by column chromatography on silica gel using a mixture of hexanes/EtOAc as eluent.

Conditions B: A toluene solution of $1(0.01 \mathrm{M})$ plus $0.5 \mathrm{~mL}$ of $\mathrm{Et}_{3} \mathrm{~N}$ was placed in a sealed tube and heated overnight at $150{ }^{\circ} \mathrm{C}$ in a silicon oil bath. After evaporation of the solvent, the crude material was purified by column chromatography on silica gel using a mixture of hexanes/EtOAc as eluent.

Conditions C: A toluene solution of $1(0.01 \mathrm{M})$ plus $0.5 \mathrm{~mL}$ of $\mathrm{MeOH}$ (or ${ }^{i} \mathrm{PrOH}$ ) was placed in a sealed tube and heated overnight at $150{ }^{\circ} \mathrm{C}$ in a silicon oil bath. After evaporation of the solvent, the crude material was purified by column chromatography on silica gel using a mixture of hexanes/EtOAc as eluent.

Conditions D: A toluene solution of $1(0.01 \mathrm{M})$ plus 1 eq of $\mathrm{AcOH}$ was placed in a sealed tube and heated overnight at $150{ }^{\circ} \mathrm{C}$ in a silicon oil bath. After evaporation of the solvent, the crude material was purified by column chromatography on silica gel using a mixture of hexanes/EtOAc as eluent.

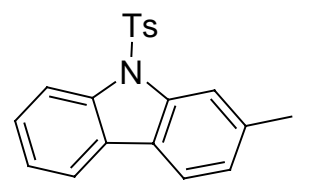

$2 \mathbf{a}$

Pale yellow powder, $40 \%$ yield (conditions B); ${ }^{1} \mathrm{H}$ NMR $\left(\mathrm{CDCl}_{3}\right) \delta: 8.29(\mathrm{~d}, J=8.4 \mathrm{~Hz}$, $1 \mathrm{H}, \operatorname{ArH}), 8.14$ (s, 1H, ArH), 7.48 (d, J= 7.5 Hz, 1H, ArH), 7.77 (d, J= $7.8 \mathrm{~Hz}, 1 \mathrm{H}$, $\operatorname{ArH}), 7.69(\mathrm{~d}, J=8.2 \mathrm{~Hz}, 2 \mathrm{H}, \operatorname{Ar} H), 7.45(\mathrm{t}, J=8.4 \mathrm{~Hz}, 1 \mathrm{H}, \operatorname{ArH}), 7.33(\mathrm{t}, J=7.5 \mathrm{~Hz}$, $1 \mathrm{H}, \operatorname{Ar} H), 7.18(\mathrm{~d}, J=7.8 \mathrm{~Hz}, 1 \mathrm{H}, \operatorname{Ar} H), 7.10(\mathrm{~d}, J=8.2,2 \mathrm{H}, \mathrm{ArH}), 2.56\left(\mathrm{~s}, 3 \mathrm{H}, \mathrm{CH}_{3}\right)$, $2.26\left(\mathrm{~s}, 3 \mathrm{H}, \mathrm{CH}_{3}\right) ;{ }^{13} \mathrm{C}$ NMR/DEPT $\left(\mathrm{CDCl}_{3}\right) \delta: 144.7(\mathrm{C}), 138.8(\mathrm{C}), 138.3(\mathrm{C}), 137.7$ $(C), 135.1(C), 129.6(2 \times C H), 126.8(C H), 126.4(2 \times C H), 125.2(C H), 124.0(C)$, $123.8(\mathrm{CH}), 120.0(C), 119.7(\mathrm{CH}), 119.6(\mathrm{CH}), 115.3(\mathrm{CH}), 115.1(\mathrm{CH}), 22.3\left(\mathrm{CH}_{3}\right)$, $21.5\left(\mathrm{CH}_{3}\right)$; MS (70 eV) m/z (\%): $335\left(\mathrm{M}^{+}, 10\right), 180(100), 152(22)$.

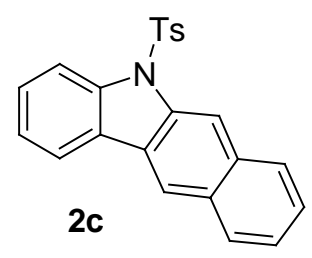

Pale yellow powder, $30 \%$ yield (conditions A); ${ }^{1} \mathrm{H} \mathrm{NMR}\left(\mathrm{CDCl}_{3}\right) \delta: 8.69(\mathrm{~d}, J=8.6 \mathrm{~Hz}$, $1 \mathrm{H}, \operatorname{Ar} H), 8.58(\mathrm{~d}, J=9.1,1 \mathrm{H}, \operatorname{Ar} H), 8.51-8.43(\mathrm{~m}, 2 \mathrm{H}, \operatorname{Ar} H), 7.99(\mathrm{~d}, J=8.4 \mathrm{~Hz}, 1 \mathrm{H}$, $\operatorname{Ar} H$ ), $7.93(\mathrm{~d}, J=9.1 \mathrm{~Hz}, 1 \mathrm{H}, \operatorname{Ar} H)$, 7.70-7.65 (m, 3H, $\operatorname{Ar} H), 7.55-7.45$ (m, 3H, $\operatorname{Ar} H$ ), 
$7.04(\mathrm{~d}, J=8.2 \mathrm{~Hz}, 2 \mathrm{H}, \mathrm{ArH}), 2.22\left(\mathrm{~s}, 3 \mathrm{H}, \mathrm{CH}_{3}\right) ;{ }^{13} \mathrm{C}$ NMR/DEPT $\left(\mathrm{CDCl}_{3}\right) \delta: 144.9(C)$, $138.2(C), 136.6(C), 135.0(C), 133.0(C), 132.0(C), 131.6(C), 130.6(C), 129.7(2 \mathrm{x}$ $C \mathrm{H}), 129.1(\mathrm{CH}), 128.6(\mathrm{CH}), 127.3(\mathrm{CH}), 126.4(2 \times C \mathrm{H}), 126.2(\mathrm{CH}), 124.9(\mathrm{CH})$, $124.3(\mathrm{CH}), 123.5(\mathrm{CH}), 122.1(\mathrm{CH}), 115.4(\mathrm{CH}), 115.0(\mathrm{CH}), 21.5\left(\mathrm{CH}_{3}\right) ; \mathrm{MS}(70 \mathrm{eV})$ $m / z(\%): 371\left(\mathrm{M}^{+}, 48\right), 216$ (100), 189 (7); HRMS $\left(\mathrm{C}_{23} \mathrm{H}_{17} \mathrm{NO}_{2} \mathrm{~S}\right)$ : calc.: 371.0980, found: 371.0977 .<smiles>CC(=O)N(C)c1ccccc1C#Cc1ccccc1</smiles>

White powder, 68\% yield; ${ }^{1} \mathrm{H}$ NMR $\left(\mathrm{CDCl}_{3}\right) \delta: 7.93(\mathrm{~d}, J=8.4 \mathrm{~Hz}, 2 \mathrm{H}, \mathrm{ArH}), 7.55$ (dd, $J=6.0,3.0 \mathrm{~Hz}, 1 \mathrm{H}, \mathrm{ArH}), 7.47-7.38(\mathrm{~m}, 3 \mathrm{H}, \mathrm{ArH}), 7.24-7.12(\mathrm{~m}, 4 \mathrm{H}, \mathrm{ArH}), 7.03-6.99$ $(\mathrm{m}, 3 \mathrm{H}, \mathrm{ArH}), 2.08\left(\mathrm{~s}, 3 \mathrm{H}, \mathrm{CH}_{3}\right), 1.89\left(\mathrm{~s}, 3 \mathrm{H}, \mathrm{CH}_{3}\right) ;{ }^{13} \mathrm{C} \mathrm{NMR} / \mathrm{DEPT}\left(\mathrm{CDCl}_{3}\right) \delta: 170.0$ (CO), $144.7(C), 138.1(C), 136.2(C), 132.9(C H), 131.7(2 \times C H), 131.4(C H), 129.8$ $(2 \times C H), 129.4(C \mathrm{H}), 128.95(C H), 128.92(2 \times C H), 128.8(C H), 128.0(2 \times C H)$, $124.0(C), 121.8(C), 95.5(C), 85.0(C), 24.6\left(C_{3}\right), 21.4\left(C_{3}\right)$; MS $(70 \mathrm{eV}) \mathrm{m} / z(\%)$ : $389\left(\mathrm{M}^{+}, 2\right), 347$ (32), $192(80), 165$ (100).

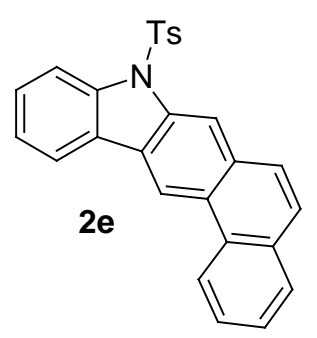

Pale yellow powder, $30 \%$ yield (conditions B); ${ }^{1} \mathrm{H}$ NMR $\left(\mathrm{CDCl}_{3}\right) \delta: 9.18(\mathrm{~s}, 1 \mathrm{H}, \mathrm{ArH})$, 8.80-8.78 (m, 2H, ArH), 7.80-7.41 (m, 10H, ArH), 7.13 (d, J= 7.6 Hz, 1H, ArH), 7.06 $(\mathrm{d}, J=8.3 \mathrm{~Hz}, 2 \mathrm{H}, \mathrm{ArH}), 2.22\left(\mathrm{~s}, 3 \mathrm{H}, \mathrm{CH}_{3}\right) ;{ }^{13} \mathrm{C}$ NMR/DEPT $\left(\mathrm{CDCl}_{3}\right) \delta: 144.9(C)$, $139.7(C), 137.6(C), 134.7(C), 131.9(C), 131.7(C), 130.8(C), 130.3(C), 130.0(C)$, $129.8(C), 129.6(2 \times C H), 128.7(C H), 128.1(C H), 127.6(C H), 127.2(C H), 126.8$ $(\mathrm{CH}), 126.53(\mathrm{CH}), 126.51(2 \times \mathrm{CH}), 124.2(\mathrm{CH}), 122.5(\mathrm{CH}), 120.4(\mathrm{CH}), 115.4(\mathrm{CH})$, $113.6(\mathrm{CH}), 113.4(\mathrm{CH}), 21.5\left(\mathrm{CH}_{3}\right) ; \mathrm{MS}(70 \mathrm{eV}) \mathrm{m} / \mathrm{z}(\%): 421\left(\mathrm{M}^{+}, 5\right), 342(40), 266$ 839), 91 (100); HRMS $\left(\mathrm{C}_{27} \mathrm{H}_{19} \mathrm{NO}_{2} \mathrm{~S}\right)$ : calc.: 421.1136 , found: 421.1140. 


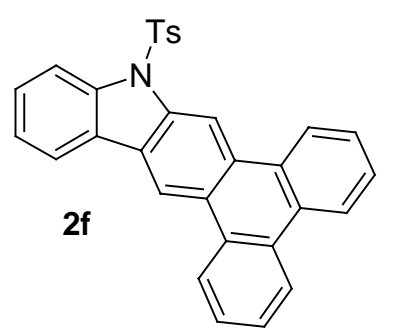

Pale yellow powder, $58 \%$ yield (conditions B) ${ }^{1} \mathrm{H}$ NMR $\left(\mathrm{CDCl}_{3}\right) \delta: 9.56(\mathrm{~s}, 1 \mathrm{H}, \mathrm{Ar} H)$, 9.06 (s, 1H, ArH), 8.85 (dd, J=8.1, 1.3 Hz, 1H, ArH), 8.71-8.68 (m, 1H, ArH), 8.38 (d, $J=8.3 \mathrm{~Hz}, 1 \mathrm{H}, \operatorname{Ar} H), 8.08(\mathrm{~d}, J=7.7 \mathrm{~Hz}, 1 \mathrm{H}, \operatorname{Ar} H), 7.77-7.63(\mathrm{~m}, 7 \mathrm{H}, \operatorname{Ar} H), 7.57-7.53$ $(\mathrm{m}, 2 \mathrm{H}, \mathrm{ArH}), 7.44$ (dt, J= 7.6, $0.9 \mathrm{~Hz}, 1 \mathrm{H}, \mathrm{ArH}), 7.04$ (d, J=8.1 Hz, 2H, ArH), 2.19 (s, $3 \mathrm{H},\left(\mathrm{CH}_{3}\right) ;{ }^{13} \mathrm{C}$ NMR/DEPT $\left(\mathrm{CDCl}_{3}\right) \delta: 144.9(\mathrm{C}), 139.8(\mathrm{C}), 138.3(\mathrm{C}), 134.7(\mathrm{C})$, $130.1(C), 129.9(C), 129.8(C), 129.7(C), 129.6(2 \times C H), 129.5(C), 128.0(C H)$, $127.51(\mathrm{CH}), 127.49(\mathrm{CH}), 127.3(\mathrm{CH}), 127.1(\mathrm{CH}), 126.7(\mathrm{C}), 126.52(2 \times \mathrm{CH}), 126.50$ $(C), 126.49(C), 124.2(C H), 123.8(C H), 123.4(C H), 123.38(C H), 123.1(C H), 120.3$ $(\mathrm{CH}), 115.5(\mathrm{CH}), 114.3(\mathrm{CH}), 108.9(\mathrm{CH}), 21.4\left(\mathrm{CH}_{3}\right)$; MS $(70 \mathrm{eV}) \mathrm{m} / \mathrm{z}(\%): 471\left(\mathrm{M}^{+}\right.$, 14), 316 (100), 291 (19); HRMS $\left(\mathrm{C}_{31} \mathrm{H}_{21} \mathrm{NO}_{2} \mathrm{~S}\right)$ : calc.: 471.1293 , found: 471.1304.

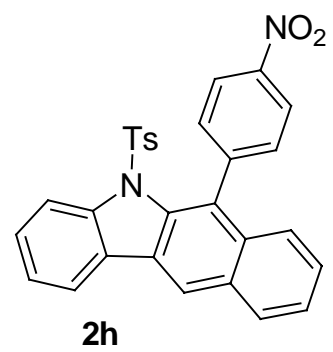

Pale yellow powder, $16 \%$ yield (conditions A); ${ }^{1} \mathrm{H} \mathrm{NMR}\left(\mathrm{CDCl}_{3}\right) \delta: 8.92(\mathrm{~s}, 1 \mathrm{H}, \mathrm{ArH})$, $8.58(\mathrm{~d}, J=2.3 \mathrm{~Hz}, 1 \mathrm{H}, \operatorname{Ar} H), 8.35$ (d, $J=8.4 \mathrm{~Hz}, 1 \mathrm{H}, \operatorname{Ar} H$ ), 8.29 (dd, $J=9.1,2.3 \mathrm{~Hz}$, $1 \mathrm{H}, \operatorname{ArH}), 8.20(\mathrm{~d}, J=9.1 \mathrm{~Hz}, 1 \mathrm{H}, \operatorname{Ar} H), 7.80(\mathrm{~d}, J=8.4 \mathrm{~Hz}, 2 \mathrm{H}, \operatorname{Ar} H), 7.66-7.63(\mathrm{~m}, 3 \mathrm{H}$, $\operatorname{ArH}), 7.48(\mathrm{t}, J=8.4 \mathrm{~Hz}, 1 \mathrm{H}, \operatorname{Ar} H), 7.40-7.37(\mathrm{~m}, 2 \mathrm{H}, \operatorname{Ar} H), 7.16(\mathrm{~d}, J=8.1 \mathrm{~Hz}, 2 \mathrm{H}$, $\operatorname{ArH}), 7.07(\mathrm{t}, J=8.1 \mathrm{~Hz}, 1 \mathrm{H}, \operatorname{ArH}), 6.65(\mathrm{~d}, J=8.4 \mathrm{~Hz}, 1 \mathrm{H}, \operatorname{ArH}), 2.29\left(\mathrm{~s}, 3 \mathrm{H},\left(\mathrm{CH}_{3}\right)\right.$;

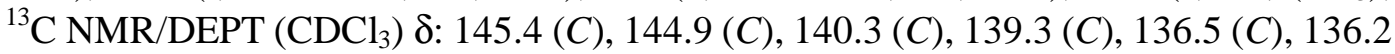
$(C), 134.9(C), 134.7(C), 129.9(2 \times C H), 129.7(2 \times C H), 129.5(2 \times C H), 129.0(C H)$, $128.8(\mathrm{CH}), 128.5(C), 126.6(2 \times C H), 126.2(C), 125.3(C), 124.1(C H), 123.4(2 \times$ $C \mathrm{H}), 123.3(\mathrm{CH}), 119.1(\mathrm{CH}), 114.7(\mathrm{CH}), 111.3(\mathrm{CH}), 21.6\left(\mathrm{CH}_{3}\right) ; \mathrm{MS}(70 \mathrm{eV}) \mathrm{m} / \mathrm{z}$ (\%): $492\left(\mathrm{M}^{+}, 12\right), 337$ (81), 290 (44), 91 (100).

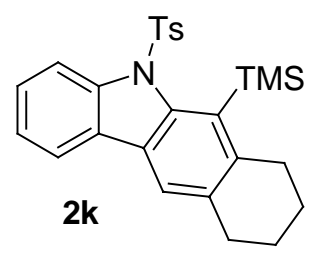

Pale yellow powder, $85 \%$ yield (conditions C) ${ }^{1} \mathrm{H}$ NMR $\left(\mathrm{CDCl}_{3}\right) \delta: 8.04(\mathrm{~d}, J=8.0 \mathrm{~Hz}$, 1H, ArH), $7.46(\mathrm{~d}, J=8.0 \mathrm{~Hz}, 1 \mathrm{H}, \operatorname{ArH}), 7.34-7.29(\mathrm{~m}, 2 \mathrm{H}, \operatorname{ArH}), 7.22-7.15(\mathrm{~m}, 2 \mathrm{H}$, $\mathrm{ArH}), 6.75-6.67(\mathrm{~m}, 3 \mathrm{H}, \mathrm{ArH}), 3.18-2.79\left(\mathrm{~m}, 4 \mathrm{H}, \mathrm{CH}_{2}\right), 1.94-1.77\left(\mathrm{~m}, 4 \mathrm{H}, \mathrm{CH}_{2}\right), 2.14$ 
(s, 3H, $\left(\mathrm{CH}_{3}\right), 0.55$ (s, 3H, $\left.\mathrm{Si}\left(\mathrm{CH}_{3}\right)_{3}\right) ;{ }^{13} \mathrm{C} \mathrm{NMR} / \mathrm{DEPT}\left(\mathrm{CDCl}_{3}\right) \delta: 145.4(\mathrm{C}), 144.6(\mathrm{C})$, $143.5(C), 141.1(C), 136.0(C), 131.7(C), 131.4(C), 130.4(C), 128.0(2 \times C H), 127.7$ $(2 \times C H), 127.0(C), 126.2(C H), 125.3(C H), 120.0(C H), 119.5(C H), 119.1(C H), 31.2$ $\left(\mathrm{CH}_{2}\right), 29.9\left(\mathrm{CH}_{2}\right), 22.8\left(\mathrm{CH}_{2}\right), 22.3\left(\mathrm{CH}_{2}\right), 21.5\left(\mathrm{CH}_{3}\right), 3.2\left(\mathrm{Si}\left(\mathrm{CH}_{3}\right)_{3}\right) ; \mathrm{MS}(70 \mathrm{eV}) \mathrm{m} / \mathrm{z}$ (\%): $447\left(\mathrm{M}^{+}, 1\right), 432$ (100), 433 (34) ; HRMS $\left(\mathrm{C}_{26} \mathrm{H}_{29} \mathrm{NO}_{2} \mathrm{SSi}\right)$ : calc. 447.1688, found:447.1696.

Thermal cyclization of. 1b and iodobenzene: A solution of. ynamide $\mathbf{1 b}(0.13 \mathrm{mmol})$, iodobenzene $(0.15 \mathrm{mmol})$ and $\mathrm{Pd}\left(\mathrm{PPh}_{3}\right)_{4}(5 \% \mathrm{mmol})$ in $\mathrm{Et}_{3} \mathrm{~N}(0.8 \mathrm{~mL})$ and toluene $(0.4$ $\mathrm{mL})$ was stirred for $10 \mathrm{~min}$ at $\mathrm{rt}$ and then $\mathrm{CuI}(2 \% \mathrm{mmol})$ was added. After heating the reaction mixture at $60{ }^{\circ} \mathrm{C}$ overnigth, the mixture was diluted with EtOAc, filtered through celite, and concentrated to dryness. The resulting crude residue was purified on silica gel column chromatography using a mixture of. hexanes/EtOAc as eluent to give $2 \mathbf{b}^{*}$ (16\% yield) and $\mathbf{2 c *}$ (8\% yield) as pale yellow powders.

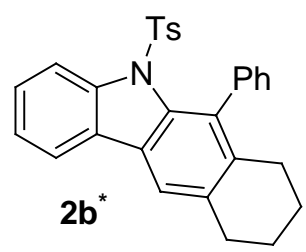

${ }^{1} \mathrm{H}$ NMR $\left(\mathrm{CDCl}_{3}\right) \delta: 8.09(\mathrm{~d}, J=7.6 \mathrm{~Hz}, 2 \mathrm{H}, \mathrm{Ar} H), 7.67(\mathrm{~d}, J=8.1 \mathrm{~Hz}, 1 \mathrm{H}, \mathrm{Ar} H), 7.51$ (s, 1H, ArH), 7.42-7.29 (m, 6H, ArH), 7.05 (d, J=8.2 Hz, 2H, ArH), 6.89 (d, J=8.2 Hz, $2 \mathrm{H}, \mathrm{ArH}), 2.98-2.95\left(\mathrm{~m}, 2 \mathrm{H}, \mathrm{CH}_{2}\right), 2.62-2.59\left(\mathrm{~m}, 2 \mathrm{H}, \mathrm{CH}_{2}\right), 2.24\left(\mathrm{~s}, 3 \mathrm{H},\left(\mathrm{CH}_{3}\right), 1.85-\right.$ $1.79(\mathrm{~m}, 2 \mathrm{H}, \mathrm{CH}), 1.74-1.69\left(\mathrm{~m}, 2 \mathrm{H}, \mathrm{CH}_{2}\right) ;{ }^{13} \mathrm{C}$ NMR/DEPT $\left(\mathrm{CDCl}_{3}\right) \delta: 143.4(\mathrm{C})$, $141.7(C), 139.3(C), 137.7(C), 136.2(C), 135.6(C), 133.8(C), 133.2(C), 130.7(2 \mathrm{x}$ $C \mathrm{H}), 129.2(C), 128.6(2 \times C H), 128.3(C), 127.6(2 \times C H), 126.8(C H), 126.6(C H)$, $126.5(2 \times C H), 124.9(C H), 119.4(C H), 119.3(C H), 119.2(C H), 30.4\left(C_{2}\right), 29.3$ $\left(\mathrm{CH}_{2}\right), 23.4\left(\mathrm{CH}_{2}\right), 22.6\left(\mathrm{CH}_{2}\right), 21.4\left(\mathrm{CH}_{3}\right)$.

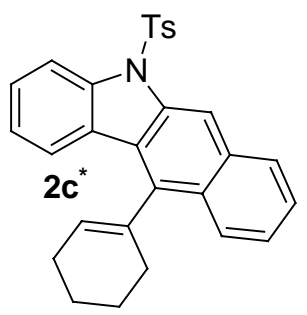

${ }^{1} \mathrm{H}$ NMR $\left(\mathrm{CDCl}_{3}\right) \delta: 8.69(\mathrm{~s}, 1 \mathrm{H}, \mathrm{Ar} H), 8.36(\mathrm{~d}, J=8.4 \mathrm{~Hz}, 1 \mathrm{H}, \mathrm{ArH}), 8.15(\mathrm{~d}, J=7.8$ $\mathrm{Hz}, 1 \mathrm{H}, \operatorname{Ar} H), 8.08(\mathrm{~d}, J=8.4 \mathrm{~Hz}, 1 \mathrm{H}, \operatorname{Ar} H), 8.04(\mathrm{~d}, J=8.1 \mathrm{~Hz}, 1 \mathrm{H}, \operatorname{Ar} H), 7.73(\mathrm{~d}, J=$ $8.0 \mathrm{~Hz}, 2 \mathrm{H}, \operatorname{ArH}), 7.56-7.47$ (m, 3H, ArH), 7.34 (t, J= 7.4 Hz, 1H, ArH), 7.07 (d, J=8.0 $\mathrm{Hz}, 2 \mathrm{H}, \mathrm{ArH}), 5.84($ broad s, $1 \mathrm{H}, \mathrm{C}=\mathrm{CH}), 2.38-2.33\left(\mathrm{~m}, 4 \mathrm{H}, \mathrm{CH}_{2}\right), 2.24\left(\mathrm{~s}, 3 \mathrm{H}, \mathrm{CH}_{3}\right)$, 1.98-1.93 (m, 4H, $\left.\mathrm{CH}_{2}\right) ;{ }^{13} \mathrm{C}$ NMR/DEPT $\left(\mathrm{CDCl}_{3}\right) \delta: 144.8(C), 140.1(C), 137.1(C)$, $136.5(C), 134.9(C), 134.8(C), 132.9(C), 129.6(2 \times \mathrm{CH}), 129.1(C), 128.6(C \mathrm{H}), 128.4$ $(\mathrm{CH}), 127.7(\mathrm{CH}), 126.6(2 \times \mathrm{CH}), 125.7(\mathrm{CH}), 125,5(\mathrm{CH}), 124.8(\mathrm{CH}), 123.9(\mathrm{CH})$, $123.1(\mathrm{CH}), 123.0(C), 114.9(\mathrm{CH}), 110.7(\mathrm{CH}), 29.4\left(\mathrm{CH}_{2}\right), 25.6\left(\mathrm{CH}_{2}\right), 23.2\left(\mathrm{CH}_{2}\right)$, $22.1\left(\mathrm{CH}_{2}\right), 21.5\left(\mathrm{CH}_{3}\right)$. 